\title{
Efficient Transmission in Multiantenna Two-Way AF Relaying Networks
}

DOI:

10.1109/TVT.2018.2791472

\section{Document Version}

Accepted author manuscript

Link to publication record in Manchester Research Explorer

\section{Citation for published version (APA):}

Yang, J., Chen, L., Lei, X., Ding, Z., Fan, P., \& Gao, X. (2018). Efficient Transmission in Multiantenna Two-Way AF Relaying Networks. IEEE Transactions on Vehicular Technology. https://doi.org/10.1109/TVT.2018.2791472

\section{Published in:}

IEEE Transactions on Vehicular Technology

\section{Citing this paper}

Please note that where the full-text provided on Manchester Research Explorer is the Author Accepted Manuscript or Proof version this may differ from the final Published version. If citing, it is advised that you check and use the publisher's definitive version.

\section{General rights}

Copyright and moral rights for the publications made accessible in the Research Explorer are retained by the authors and/or other copyright owners and it is a condition of accessing publications that users recognise and abide by the legal requirements associated with these rights.

\section{Takedown policy}

If you believe that this document breaches copyright please refer to the University of Manchester's Takedown Procedures [http://man.ac.uk/04Y6Bo] or contact uml.scholarlycommunications@manchester.ac.uk providing relevant details, so we can investigate your claim.

\section{OPEN ACCESS}




\title{
Efficient Transmission in Multi-Antenna Two-Way AF Relaying Networks
}

\author{
Jing Yang, Member, IEEE, Lei Chen, Student Member, IEEE, Xianfu Lei, Senior Member, IEEE, \\ Zhiguo Ding, Senior Member, IEEE, Pingzhi Fan, Fellow, IEEE, and Xiqi Gao, Fellow, IEEE
}

\begin{abstract}
In this paper, an efficient transmission scheme, termed the joint antenna selection and data exchange (AS-DE) scheme, is proposed for a two-way amplify-and-forward relaying network, where two single-antenna source terminals exchange information via a multi-antenna relay station. For the proposed scheme, the best antenna at the relay for each source terminal is first selected separately, following the max-max scheme. Then, from the set of the previously selected antennas, either one antenna is selected, in a similar fashion as well-known maxmin and max-sum schemes, or two antennas exchange their respective received signals, which are then coded, amplified and broadcasted to the source and destination terminals. Tight lower and upper bounds on the outage probability (OP) for the proposed scheme have been derived assuming independent and identically distributed Rayleigh fading channels. Furthermore, our analysis reveals that the proposed joint AS-DE scheme can achieve full diversity. Finally, it is shown that under the same resource constraints, i.e., in terms of the number of the utilized time slots and transmit power, the proposed joint ASDE scheme outperforms the max-min, the max-sum and the max-max schemes. Extensive numerical results accompanied with computer simulations, are further provided to validate the developed analytical results.
\end{abstract}

Index Terms-Two-way relaying networks, outage probabilities, antenna selection, max-min, max-sum, max-max.

Copyright (C) 2015 IEEE. Personal use of this material is permitted. However, permission to use this material for any other purposes must be obtained from the IEEE by sending a request to pubs-permissions @ieee.org.

Manuscript received Jan. 22, 2015; revised Apr. 6, 2015, Jul. 1, 2015, Jan. 21, 2016 and Sep. 18, 2016; accepted Dec. 20, 2017. This work of J. Yang was supported in part by National Natural Science Foundation of China under Grant 61472343, and China Postdoctoral Science Foundation (Grant No. 2014M560374). The work of X. Lei was supported in part by the Sichuan International Science and Technology Cooperation Project under Grant 2017HH0035, in part by the National Natural Science Foundation of China under Grant 61501382, and in part by the open research fund of the National Mobile Communications Research Laboratory, Southeast University, under Grant 2017D15. The work of Z. Ding was supported by the UK EPSRC under grant number EP/N005597/1 and by H2020-MSCA-RISE-2015 under grant number 690750. The work of P. Fan was supported by NSFC No.61471302. The work of X. Gao was supported by National Natural Science Foundation of China under Grants 61320106003, 61471113, 61521061 and 61631018, the China High-Tech 863 Plan under Grants 2015AA01A701 and 2014AA01A704, National Science and Technology Major Project of China under Grant 2017ZX03001002-004, and the Huawei Cooperation Project. The review of this paper was coordinated by Prof. Jayaweera Sudharman.

This work was presented in part at 2012 International Conference on Wireless Communications and Signal Processing (WCSP 2012), Nanjing, China, Oct., 2012.

J. Yang and L. Chen are with Yangzhou University, Yangzhou, China (e-mails: jingyang@yzu.edu.cn, leichen092@163.com). J. Yang is also with Southeast University, Nanjing, China.

X. Gao is with Southeast University, Nanjing, China (e-mail: xqgao@seu.edu.cn).

X. Lei and P. Fan are with Southwest Jiaotong University, China (e-mails: xflei81@gmail.com,pzfan@swjtu.edu.cn).

Z. Ding is with Lancaster University, UK (e-mail: z.ding @lancaster.ac.uk).

\section{INTRODUCTION}

Recently, two-way relaying networks (TWRNs) have been envisioned as a promising transmission technology to significantly improve the reliability and transmission rate of wireless systems [1], [2]. The performance of TWRNs can be further improved by integrating multiple-input multiple-output (MIMO) transmission technology [3]-[5]. Antenna selection (AS), i.e., optimally choosing a subset of the available antennas, is an attractive low-cost and low-complexity technique, but still retains many of the advantages of conventional MIMO systems [6]. In the open technical literature, three antenna selection schemes for MIMO amplify-and-forward (AF) and decodeand-forward (DF) TWRNs have been proposed, namely the max-min [7], [8], the max-sum [9] and the max-max schemes [10], [11].

The performance achieved by such schemes has been assessed in several past research works. For example, the outage probability (OP) performance of the max-min and the maxsum schemes has been evaluated in [7]-[9]. These works have shown that both schemes can achieve full diversity. In [10], antenna selection in a DF relaying network based on the maxmax scheme was investigated, assuming that decoding at the relay is error-free. In [11], the so-called double-max scheme was proposed. In that work, relay selection based on the maxmax scheme was addressed, assuming the use of an error-free decoding relay.

Motivation: For the purpose of illustration, consider two single-antenna sources $T_{1}$ and $T_{2}$ exchanging information via a relay station $R$ which is equipped with $N=3$ antennas, denoted by antenna $R_{1}$, antenna $R_{2}$ and antenna $R_{3}$, respectively. For example, let the channel gains from $T_{1}$ and $T_{2}$ to $R$ at a given time instant be $h=\left\{h_{1}, h_{2}, h_{3}\right\}=\{0.35,0.46,0.59\}$ and $g=\left\{g_{1}, g_{2}, g_{3}\right\}=\{0.72,0.54,0.32\}$, respectively. According to the max-min scheme, the best antenna at the relay is selected to maximize the end-to-end signal-to-noise ratio of the worse source [7], [8]. In this example, the antenna antenna $R_{2}$ will be chosen with $h_{2}=0.46$ and $g_{2}=0.54$. However, it can be observed that the links having the largest channel gains, i.e., $h_{3}=0.59$ and $g_{1}=0.72$, have not been utilized.

When the max-sum scheme is utilized, the best antenna at the relay is selected to maximize the sum-rate [9]. In the considered test case, the antenna $R_{1}$ will be chosen with $h_{1}=0.35$ and $g_{1}=0.72$. However, as it can be observed this method does not exploit the channel coefficient $h_{3}=0.59$, i.e., the maximum channel gain in all $h_{i} \mathrm{~s}, i \in\{1,2,3\}$. 
The max-max scheme selects at a given time instance either one or two antennas at the relay, corresponding to the maximum channel coefficients [10], [11]. If the antenna indices are the same, one antenna is selected, otherwise two antennas are selected. In the previously described example, two antennas are selected, namely the antenna $R_{1}$ and antenna $R_{3}$ corresponding to the links with $h_{3}=0.59$ and $g_{1}=0.72$. Consider, however, the following data transmission scenario from $T_{1}$ to $T_{2}$. Specifically, assume that information flows from the links $T_{1} \rightarrow R_{1}, R_{1} \rightarrow T_{2}$ and $T_{1} \rightarrow R_{3}, R_{3} \rightarrow T_{2}$, characterized by channel gains $h_{1}=0.35, g_{1}=0.72$ and $h_{3}=0.59, g_{3}=0.32$, respectively. As can be observed, during the transmission through the antenna $R_{1}$, link $T_{1} \rightarrow R_{1}$ experiences the worse channel conditions since $h_{1}$ is the minimum channel coefficient in $h_{i}$ s. On the other hand, link $R_{1} \rightarrow T_{2}$ experiences the best channel conditions because $g_{1}$ is the maximum channel coefficient in $g_{i}$ s. Similar findings can be found when transmission through the links $T_{1} \rightarrow R_{3}$, $R_{3} \rightarrow T_{2}$ is considered. In such scenarios, the combinations of "small-maximum" and "maximum-small" channel coefficients result in a small received end-to-end (e2e) SNR at $T_{2}$. Note that when one antenna is selected, i.e., when the selected antennas' indices are identical, data transmission will exploit the best links in an optimal way. In such a case, the max-max scheme exhibits the best performance. However, this is a small probability event.

Motivated by this key observation, in this paper, an efficient transmission scheme which can exploit the unutilized links characterized by the best channel coefficients, termed the joint antenna selection and data exchange (AS-DE) scheme, is proposed for multi-antenna AF TWRNs. The key idea in the joint AS-DE scheme is to combine max-max antenna selection scheme along with data exchange to transmit data through the links characterized by "maximum-maximum" and "smallsmall" channel coefficients. Consequently, the joint AS-DE scheme outperforms the max-min, the max-sum and the maxmax schemes because its e2e SNR is significantly larger than that achieved by the aforementioned AS schemes. It should be emphasized that the previously reported works on the maxmax scheme, such as those presented in [10], [11], ignore the possible transmission error due to the aforementioned "smallmaximum" and "maximum-small" combinations of channel coefficients, since they consider DF relaying networks and assume decoding at the relay is error-free.

The performance of the joint AS-DE scheme is assessed by deriving the tight upper and lower bounds on the e2e OP, assuming Rayleigh fading conditions. The tightness of the newly derived bounds is verified by means of computer simulation. Extensive numerical results are further presented revealing that the joint AS-DE scheme can achieve full diversity. In addition, it is shown that under the same resource consumption constraints, such as in terms of the utilized time slots and transmit power, the joint AS-DE scheme also outperforms the existing max-min, max-sum and max-max schemes.

The remainder of this paper is organized as follows: Section II presents the system model and the joint AS-DE scheme. Section III investigates the OP and diversity gain performance for the joint AS-DE scheme. Numerical and simulation results are presented in Section IV. Finally, Section V concludes the paper.

Notation: $\mathbb{E}\{\cdot\}$ and $\mathbf{I}_{M}$ denote the expectation operation and an $M \times M$ identity matrix, respectively. $K_{v}(\cdot)$ and $\operatorname{Ei}(\cdot)$ denote the $v$ order modified Bessel function of the second kind [12, Eq. (8.407)] and the Exponential integral function [12, Eq. (8.211)], respectively. The notations $\mathcal{C N}\left(0, \sigma^{2}\right), f_{X}(\cdot)$ and $F_{X}(\cdot)$ represent a circularly symmetric complex Gaussian random variable (RV) with zero mean and variance $\sigma^{2}$, the probability density function (PDF) and cumulative distribution function $(\mathrm{CDF})$ of $\mathrm{RV} \mathrm{X}$, respectively. $\operatorname{Pr}(\cdot)$ returns the probability.

\section{System Model And the Proposed Joint AS-DE SCHEME}

In this section, the system model and the joint AS-DE scheme are introduced.

\section{A. System Model}

Consider a TWRN, where two single-antenna source terminals $T_{1}$ and $T_{2}$ exchange information by using an AF relay station $R$ equipped with $N \geq 2$ antennas. Assume that all the links experience independent and identically distributed (i.i.d.) Rayleigh fading, following $\mathcal{C N}(0, \Omega)$, and channels are reciprocal. Assume that the $i$-th antenna $R_{i}$ is selected to help the communication between $T_{1}$ and $T_{2}$. The whole communication takes place in two times slots. In the first time slot, $T_{1}$ and $T_{2}$ transmit their signals to $R$. The received signal at the antenna $R_{i}$ after $M$ successive symbol durations can be written as

$$
\mathbf{y}_{i}=\sqrt{P} h_{i} \mathbf{s}_{1}+\sqrt{P} g_{i} \mathbf{s}_{2}+\mathbf{n}_{i},
$$

where $\mathbf{s}_{j}=\left[s_{j}(1), \cdots, s_{j}(M)\right]^{T}, j=1,2$, denotes the transmitted symbol of $T_{j}$ with $\mathbb{E}\left[\mathbf{s}_{j} \mathbf{s}_{j}^{\dagger}\right]=\mathbf{I}_{M}, P$ is the transmit power of $T_{j}, h_{i}$ and $g_{i}$ denote the channel coefficients between $T_{1}$ and antenna $R_{i}$, and between $T_{2}$ and the antenna $R_{i}$, respectively, $\mathbf{n}_{i} \sim \mathcal{C N}\left(0, N_{0} \mathbf{I}_{M}\right)$ represents additive gaussian white noise (AWGN) at the antenna $R_{i}$.

In the second time slot, the selected antenna $R_{i}$ amplifies its received signal with gain $\alpha$ and then broadcasts it to $T_{j}$. The received signals at $T_{1}$ and $T_{2}$ are given by

$$
\mathbf{y}_{T_{1}}=\sqrt{P_{r}} h_{i} \alpha \mathbf{y}_{i}+\mathbf{n}_{T_{1}} \text {, and } \mathbf{y}_{T_{2}}=\sqrt{P_{r}} g_{i} \alpha \mathbf{y}_{i}+\mathbf{n}_{T_{2}},
$$

respectively, where $P_{r}$ denotes the transmit power of the $i$-th antenna at $R$ and $\left.\mathbf{n}_{T_{j}} \sim \mathcal{C N}\left(0, N_{0} \mathbf{I}_{M}\right)\right)$ is AWGN at terminal $T_{j}$. Assuming that fixed gain relaying is used, the amplification factor is expressed as [1], [13],

$$
\alpha=\sqrt{\frac{1}{2 P \Omega+N_{0}}} .
$$

After the self-interference cancellation is performed, assuming $P_{r}=2 P$, the received SNR at $T_{1}$ and $T_{2}$ via the help of the antenna $R_{i}$ is given as [13],

$$
\gamma_{i}^{T_{1}}=\frac{2 \gamma_{i}^{l} \gamma_{i}^{r}}{2 \gamma_{i}^{l}+c}, \text { and } \gamma_{i}^{T_{2}}=\frac{2 \gamma_{i}^{l} \gamma_{i}^{r}}{2 \gamma_{i}^{r}+c},
$$


where $\gamma_{i}^{l}=P\left|h_{i}\right|^{2} / N_{0}, \gamma_{i}^{r}=P\left|g_{i}\right|^{2} / N_{0}, \bar{\gamma}=P \Omega / N_{0}$, and $c=2 \bar{\gamma}+1$.

In the following, the three conventional AS schemes, i.e., the max-min, the max-sum and the max-max schemes, are introduced.

- In the max-min scheme, the $i^{*}$-th antenna is selected according to [7], [8],

$$
i^{*}=\arg \max _{1 \leq i \leq N} \min \left(\gamma_{i}^{T_{1}}, \gamma_{i}^{T_{2}}\right) .
$$

- In the max-sum scheme, the selected antenna $i^{*}$ follows [9]

$$
i^{*}=\arg \max _{1 \leq i \leq N}\left(\left(1+\gamma_{i}^{T_{1}}\right)\left(1+\gamma_{i}^{T_{2}}\right)\right) .
$$

From (4) and (5), it can be observed that only one antenna can be selected for relaying between $T_{1}$ and $T_{2}$ in both the max-min and max-sum schemes.

- In the max-max scheme, the $l^{*}$-th and $r^{*}$-th antennas are selected according to [10], [11],

$$
l^{*}=\arg \max _{1 \leq i \leq N} h_{i}, \text { and } r^{*}=\arg \max _{1 \leq j \leq N} g_{j} .
$$

From (6), it can be observed that if the antenna indices $l^{*}=r^{*}$, only one antenna can be used for relaying between $T_{1}$ and $T_{2}$ in the max-max scheme. Otherwise, two antennas can be used. Besides, it can be seen that the selected $l^{*}$-th and $r^{*}$-th antennas have the largest channel gain to $T_{1}$ and $T_{2}$, respectively.

\section{B. The Joint AS-DE Scheme}

The proposed joint AS-DE scheme includes two procedures, i.e., antenna selection and data exchange. Antenna selection is performed based on the max-max scheme in a similar fashion as in (6). During the data exchange phase, the $l^{*}$-th antenna at $R$ transmits its signal $\mathbf{y}_{l^{*}}$ to the $r^{*}$-th antenna at $R$, and the $r^{*}$ th antenna at $R$ transmits its signal $\mathbf{y}_{r^{*}}$ to the $l^{*}$-th antenna at $R$. We note that data exchange is started only when two antennas are selected, i.e., the antenna indices $l^{*} \neq r^{*}$. The whole communication takes place in two times slots.

Let us now rearrange $h_{i}$ and $g_{i}, i=1, \cdots, N$, in an ascending order. We define the channel coefficients $h_{(i)}$ and $g_{(i)}$, respectively, such that $h_{(1)} \leq h_{(2)} \leq \cdots \leq h_{(N)}=h_{l^{*}}$ and $g_{(1)} \leq g_{(2)} \leq \cdots \leq g_{(N)}=g_{r^{*}}$. Note that $h_{l^{*}}$ and $g_{r^{*}}$ are the $N$-th largest channel gain in $h_{i} \mathrm{~s}$ and $g_{i} \mathrm{~s}$, respectively, but $h_{r^{*}}$ and $g_{l^{*}}$ are the $w$-th largest channel gain, $1 \leq w \leq N-1$, and $q$-th largest, $1 \leq q \leq N-1$, in $h_{i}$ s and $g_{i}$ s, respectively. We denote $\gamma_{(N)}^{l}=P\left|h_{l^{*}}\right|^{2} / N_{0}, \gamma_{(w)}^{l}=P\left|h_{r^{*}}\right|^{2} / N_{0}$, $\gamma_{(N)}^{r}=P\left|g_{r^{*}}\right|^{2} / N_{0}$, and $\gamma_{(q)}^{r}=P\left|g_{l^{*}}\right|^{2} / N_{0}$.

In the following, data transmission in the joint AS-DE scheme is presented when either one or two antennas are selected.

1) If One Antenna is Selected: In this case, the antenna indices are the same, i.e., $l^{*}=r^{*}$. In the first time slot, $T_{1}$ and $T_{2}$ broadcast their information to $R$. In the second time slot, the selected antenna amplifies its received signal in the first time slot by a gain $\alpha$, and broadcasts to $T_{1}$ and $T_{2}$ with full power $P_{r}=2 P$.
Similar to (3), the received SNR at $T_{2}$ can be obtained as

$$
\gamma_{1, T_{2}}=\frac{2 \gamma_{(N)}^{l} \gamma_{(N)}^{r}}{2 \gamma_{(N)}^{r}+c}
$$

2) If Two Antennas are Selected: In this case, the antenna indices are different, i.e., $l^{*} \neq r^{*}$. In the first time slot, the received signals at $R$ via the antennas $l^{*}$ and $r^{*}$, which have been selected based on (6), are given by

$$
\mathbf{y}_{l^{*}}=\sqrt{P} h_{l^{*}} \mathbf{s}_{1}+\sqrt{P} g_{l^{*}} \mathbf{s}_{2}+\mathbf{n}_{l^{*}},
$$

and

$$
\mathbf{y}_{r^{*}}=\sqrt{P} h_{r^{*}} \mathbf{s}_{1}+\sqrt{P} g_{r^{*}} \mathbf{s}_{2}+\mathbf{n}_{r^{*}},
$$

respectively. The $\mathbf{n}_{l^{*}}, \mathbf{n}_{r^{*}} \sim \mathcal{C N}\left(0, N_{0} \mathbf{I}_{M}\right)$ represent the AWGN at the $l^{*}$-th and $r^{*}$-th antennas, respectively.

Following [13], the durations of both time slots are considered to be the same. Data exchange between the $l^{*}$-th and $r^{*}$-th antennas occurs in the second time slot. Then, the selected $l^{*}$ th antenna transmits its signal $y_{l^{*}}$ to the $r^{*}$-th antenna, and the $r^{*}$-th antenna transmits its signal $y_{r^{*}}$ to the $l^{*}$-th antenna. In the same time slot, the $l^{*}$-th and $r^{*}$-th antennas process $y_{r^{*}}$ and $y_{l^{*}}$ to generate the space time coded symbol $\mathbf{x}_{l^{*}}$ and $\mathbf{x}_{r^{*}}$, respectively. The transmitted signals $\mathbf{x}_{l^{*}}$ and $\mathbf{x}_{r^{*}}$ are designed to be linear functions of $\mathbf{y}_{r^{*}}$ and $\mathbf{y}_{l^{*}}$ and their conjugates, namely [13]-[16],

$$
\mathbf{x}_{l^{*}}=\mathbf{A}_{l^{*}} \mathbf{y}_{r^{*}}+\mathbf{B}_{l^{*}} \mathbf{y}_{r^{*}}^{*} \text {, and } \mathbf{x}_{r^{*}}=\mathbf{A}_{r^{*}} \mathbf{y}_{l^{*}}+\mathbf{B}_{r^{*}} \mathbf{y}_{l^{*}}^{*},
$$

where $\mathbf{A}_{p}$ and $\mathbf{B}_{p}, p \in\left\{l^{*}, r^{*}\right\}$, are $M \times M$ precoding matrices, designed using guidelines for the construction of distributed space-time coding schemes, and $\mathbf{y}_{p}^{*}$ denotes the conjugate of $\mathbf{y}_{p}$. For simplicity, in this paper, we consider $M=2$, and the orthogonal matrices are used at the two selected antennas as in [16], namely

$$
\mathbf{A}_{l^{*}}=\mathbf{I}_{2}, \mathbf{B}_{l^{*}}=\mathbf{0}_{2}, \mathbf{A}_{r^{*}}=\mathbf{0}_{2}, \mathbf{B}_{r^{*}}=\left(\begin{array}{cc}
0 & -1 \\
1 & 0
\end{array}\right)
$$

Therefore, (8) becomes

$$
\mathbf{x}_{l^{*}}=\mathbf{A}_{l^{*}}\left(\sqrt{P} h_{r^{*}} \mathbf{s}_{1}+\sqrt{P} g_{r^{*}} \mathbf{S}_{2}+\mathbf{n}_{r^{*}}\right),
$$

and

$$
\mathbf{x}_{r^{*}}=\mathbf{B}_{r^{*}}\left(\sqrt{P} h_{l^{*}} \mathbf{s}_{1}+\sqrt{P} g_{l^{*}} \mathbf{s}_{2}+\mathbf{n}_{l^{*}}\right)^{*} .
$$

Then, the $l^{*}$-th and $r^{*}$-th antennas broadcast $\mathbf{x}_{l^{*}}$ and $\mathbf{x}_{r^{*}}$ after amplification, respectively, each with half power $P_{r} / 2=$ $P$. Let $\widetilde{\mathbf{y}}_{T_{2}}$ denote the received signals after self-interference cancellation at $T_{2}$, given by

$$
\begin{aligned}
\widetilde{\mathbf{y}}_{T_{2}}= & \sqrt{\frac{P_{r}}{2}} g_{r^{*}} \alpha^{\prime} \mathbf{B}_{r^{*}}\left(\sqrt{P} h_{l^{*}} \mathbf{s}_{1}+\mathbf{n}_{l^{*}}\right)^{*} \\
& +\sqrt{\frac{P_{r}}{2}} g_{l^{*}} \alpha^{\prime} \mathbf{A}_{l^{*}}\left(\sqrt{P} h_{r^{*}} \mathbf{s}_{1}+\mathbf{n}_{r^{*}}\right)+\mathbf{n}_{T_{2}} .
\end{aligned}
$$

The average transmit power at each antenna is constrained to

$$
\mathbb{E}\left\{\left\|\alpha^{\prime} \mathbf{x}_{p}\right\|_{F}^{2}\right\}=1 \text {. }
$$


When orthogonal matrices in (9) are employed, $\alpha^{\prime}=\alpha / \sqrt{2}$, and the received SNR at $T_{2}$ can be obtained, based on (10), as follows

$$
\begin{aligned}
& \gamma_{2, T_{2}}^{\mathrm{ASDE}} \\
& =\frac{\alpha^{2} P P\left|h_{l^{*}}\right|^{2}\left|g_{r^{*}}\right|^{2}\left\|\mathbf{B}_{r^{*}}\right\|^{2}+\alpha^{2} P P\left|h_{r^{*}}\right|^{2}\left|g_{l^{*}}\right|^{2}\left\|\mathbf{A}_{l^{*}}\right\|^{2}}{\alpha^{2} P\left|g_{r^{*}}\right|^{2}\left\|\mathbf{B}_{r^{*}}\right\|^{2} N_{0}+\alpha^{2} P\left|g_{l^{*}}\right|^{2}\left\|\mathbf{A}_{l^{*}}\right\|^{2} N_{0}+2 N_{0}} .
\end{aligned}
$$

Furthermore, (12) can be re-expressed as

$$
\gamma_{2, T_{2}}^{\mathrm{ASSE}}=\frac{\gamma_{(N)}^{r} \gamma_{(N)}^{l}+\gamma_{(q)}^{r} \gamma_{(w)}^{l}}{\gamma_{(q)}^{r}+\gamma_{(N)}^{r}+c} .
$$

Eq. (13) can be upper- and lower-bounded as

$$
\gamma_{T_{2}, \mathrm{ub}}^{\mathrm{ASDE}} \geq \gamma_{2, T_{2}}^{\mathrm{ASDE}} \geq \gamma_{T_{2}, \mathrm{lb}}^{\mathrm{ASDE}},
$$

where

$$
\begin{aligned}
\gamma_{T_{2}, \mathrm{lb}}^{\mathrm{ASDE}} & =\frac{\gamma_{(N)}^{r} \gamma_{(N)}^{l}}{\gamma_{(q)}^{r}+\gamma_{(N)}^{r}+c}, \\
\gamma_{T_{2}, \mathrm{ub}}^{\mathrm{ASDE}} & =\frac{\gamma_{(N)}^{l}\left(\gamma_{(q)}^{r}+\gamma_{(N)}^{r}\right)}{\gamma_{(q)}^{r}+\gamma_{(N)}^{r}+c} .
\end{aligned}
$$

To compare the joint AS-DE scheme with the max-max scheme, here, we present the received SNR at $T_{2}$ in the maxmax scheme. In the max-max scheme, since it does not utilize the "data exchange", in the second time slot, the transmitted signal $\mathbf{x}_{l^{*}}^{\prime}$ at the $l^{*}$-th antenna and the transmitted signal $\mathbf{x}_{r^{*}}^{\prime}$ at the $r^{*}$-th antenna are given as

$$
\mathbf{x}_{l^{*}}^{\prime}=\mathbf{A}_{l^{*}}\left(\sqrt{P} h_{l^{*}} \mathbf{s}_{1}+\sqrt{P} g_{l^{*}} \mathbf{S}_{2}+\mathbf{n}_{l^{*}}\right),
$$

and

$$
\mathbf{x}_{r^{*}}^{\prime}=\mathbf{B}_{r^{*}}\left(\sqrt{P} h_{r^{*}} \mathbf{s}_{1}+\sqrt{P} g_{r^{*}} \mathbf{S}_{2}+\mathbf{n}_{r^{*}}\right)^{*}
$$

respectively.

Following similar arguments as to (13), the received SNR at $T_{2}$ in the max-max scheme can be obtained as

$$
\gamma_{2, T_{2}}^{\operatorname{MaxMax}}=\frac{\gamma_{(q)}^{r} \gamma_{(N)}^{l}+\gamma_{(N)}^{r} \gamma_{(w)}^{l}}{\gamma_{(q)}^{r}+\gamma_{(N)}^{r}+c} .
$$

Remark 1: Comparing (13) with (17), it can be seen that the difference between the two SNR results is in their numerators. In the numerator of (13), there exists the "maximummaximum", i.e. $\gamma_{(N)}^{r} \gamma_{(N)}^{l}$, and "small-small", i.e., $\gamma_{(q)}^{r} \gamma_{(w)}^{l}$, channel coefficient combinations for the joint AS-DE scheme, but "small-maximum" and "maximum-small", i.e., $\gamma_{(q)}^{r} \gamma_{(N)}^{l}$ and $\gamma_{(N)}^{r} \gamma_{(w)}^{l}$, link combinations in (17) for the max-max scheme. This is because both $\gamma_{(N)}^{r}$ and $\gamma_{(N)}^{l}$ are the maximal effective channel gain, due to the fact that $g_{r^{*}}$ and $h_{l^{*}}$ are the $N$-th maximum among $g_{i} \mathrm{~s}$ and $h_{i} \mathrm{~s}$; and, $\gamma_{(q)}^{r}$ and $\gamma_{(w)}^{l}$ are small, due to the fact that $g_{l^{*}}$ and $h_{r^{*}}$ are the $q$-th maximum and $w$-th maximum in $g_{i} \mathrm{~s}$ and $h_{i} \mathrm{~s}$, respectively. The "maximum-maximum" and "small-small" link combinations in the joint AS-DE scheme, result in a larger received SNR than in the case of the max-max scheme, where the "smallmaximum" and "maximum-small" link combinations are used. Although the max-max scheme exploits the best links with the largest channel gains, it does not utilize them in the best manner. Recalling the example described in the introduction section, one can find that the max-min and the max-sum schemes may not exploit the best links. However, in the joint AS-DE scheme data transmission from $T_{1}$ to $T_{2}$ uses the links $T_{1} \rightarrow R_{3}, R_{1} \rightarrow T_{2}$ and $T_{1} \rightarrow R_{1}, R_{3} \rightarrow T_{2}$, having gains $h_{3}=0.59, g_{1}=0.72$ and $h_{1}=0.35, g_{3}=0.32$, respectively. From this and (13), we conclude that the joint AS-DE scheme exploits the strong channel links in the best manner, by utilizing "maximum-maximum" and "small-small" channel coefficients combinations. Because of this, it outperforms the max-max, the max-min and the max-sum schemes.

Remark 2: Despite the fact that the joint AS-DE scheme outperforms the conventional AS schemes, it requires a second RF chain for its practical implementation. Moreover, its baseband implementation is more complicated than the one of conventional schemes, as it requires data exchange between the selected antennas. Recently, novel MIMO transmission schemes have been reported, such as spatial modulation and MIMO electronically steerable passive array radiator (ESPAR) [17]-[19]. Such schemes can minimize complexity and the costs while attaining the advantages of the MIMO system.

Remark 3: Hereafter some issues regarding the implementation of the data exchange phase of the joint AS-DE scheme are discussed. Such a scheme can be implemented in an efficient manner in baseband, by employing digital hardware, instead of exchanging analog signals between antennas. Specialized devices, such as digital signal processors (DSP) or field programmable gate arrays (FPGA) can be used to this purpose. Such devices are equipped with specialized direct memory access (DMA) controllers, thus rendering them capable of transferring large amounts of data, stored in buffers. Sophisticated techniques, such as multiple buffering, can be also employed to increase the efficiency of data transmission. Data exchange between two antennas can be implemented without significant computational complexity by exchanging the contents of their corresponding buffers. For a given hardware platform, one can perform such a task in an optimzied way, i.e., by minimizing the number of the required clock cycles.

\section{Performance Analysis}

In this section, the OP performance at $T_{2}$ will be analyzed. The performance analysis at $T_{1}$ can be obtained in a similar fashion and thus mathematical derivations are omitted for brevity. For writing simplicity, some definitions are given as follows.

Definition 1:

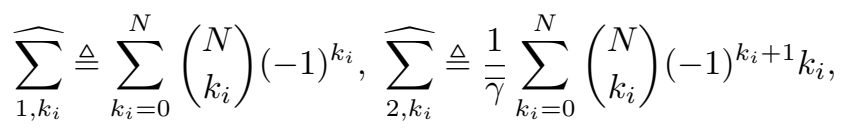

and

$$
\begin{gathered}
\widehat{\sum_{3, k_{i}, k_{j}}}=\frac{1}{\bar{\gamma}^{2}} \frac{N !}{(q-1) !(N-1-q) !} \sum_{k_{i}=0}^{q-1} \sum_{k_{j}=0}^{N-1-q}\left(\begin{array}{c}
q-1 \\
k_{i}
\end{array}\right) \\
\times\left(\begin{array}{c}
N-q-1 \\
k_{j}
\end{array}\right)(-1)^{k_{i}+k_{j}} .
\end{gathered}
$$


The distributions of $\gamma_{1, T_{2}}$ and $\gamma_{2, T_{2}}^{\mathrm{ASDE}}$ will be firstly presented in the following theorems, which lay the foundation for performance analysis.

\section{A. Distribution of the received SNR}

Theorem 1: When one antenna is selected for relaying between $T_{1}$ and $T_{2}$, i.e., the antenna indices $l^{*}=r^{*}$, the CDF of the received SNR at terminal $T_{2}$, i.e., $\gamma_{1, T_{2}}$, can be expressed in closed-form as

$F_{\gamma_{1, T_{2}}}(z)=1+\widehat{\sum_{1, k_{1} \neq 0}} \widehat{\sum_{2, k_{2} \neq 0}} \sqrt{\frac{2 k_{1} z c}{k_{2}}} e^{-\frac{k_{1}}{\bar{\gamma}} z} K_{1}\left(\frac{\sqrt{2 k_{1} k_{2} z c}}{\bar{\gamma}}\right)$.

Proof: See Appendix A.

Theorem 2: When two antennas are selected for relaying between $T_{1}$ and $T_{2}$, i.e., the antenna indices $l^{*} \neq r^{*}$, the CDF of the upper bound on $\gamma_{2, T_{2}}^{\mathrm{ASDE}}$, i.e., $F_{\gamma_{T_{2}, \text { ub }}^{\mathrm{ASDE}}}(z)$, is given as

$$
F_{\gamma_{T_{2}, \mathrm{ub}}^{\mathrm{ASD}}}(z)= \begin{cases}\mathcal{L}_{1}, & a=b \\ \mathcal{L}_{2}, & a \neq b\end{cases}
$$

where

$$
a=\frac{k_{2}+1}{\bar{\gamma}}, b=\frac{N+k_{1}-q-k_{2}}{\bar{\gamma}},
$$

$\mathcal{L}_{1}=\widehat{\sum_{3, k_{1}, k_{2}}}\left[\frac{1}{a(a+b)}+\sum_{1, k_{3} \neq 0} \frac{k_{3} c z}{a \bar{\gamma}} e^{-\frac{k_{3} z}{\bar{\gamma}}} K_{2}\left(2 \sqrt{\frac{k_{3} a c z}{\bar{\gamma}}}\right)\right]$,

and

$$
\begin{aligned}
\mathcal{L}_{2}=\widehat{\sum_{3, k_{1}, k_{2}}} & {\left[\frac{1}{a(a+b)}+\sum_{1, k_{3} \neq 0} \frac{2 c e^{-\frac{k_{3} z}{\bar{\gamma}}}}{b-a} \sqrt{\frac{k_{3} z}{a c \bar{\gamma}}} K_{1}\left(2 \sqrt{\frac{k_{3} a c z}{\bar{\gamma}}}\right)\right.} \\
& \left.-\sqrt{\frac{2 k_{3} z}{(a+b) c \bar{\gamma}}} K_{1}\left(\sqrt{\frac{2 k_{3}(a+b) c z}{\bar{\gamma}}}\right)\right] .
\end{aligned}
$$

Proof: See Appendix B.

Theorem 3: When two antennas are selected, i.e., $l^{*} \neq r^{*}$, the $\mathrm{CDF}$ of the lower bound on $\gamma_{2, T_{2}}^{\mathrm{ASDE}}$, i.e., $F_{\gamma_{T_{2}, \mathrm{bl}}^{\mathrm{ASDE}}}(z)$, is given as,

$$
F_{\gamma_{T_{2}, \mathrm{Lb}}^{\mathrm{ASE}}}(z)=\widehat{\sum_{3, k_{1}, k_{2} 1, k_{3}}} \widehat{\sum}\left(\int_{0}^{1} e^{-\frac{k_{3} z}{\gamma \zeta}} \mathcal{A}_{1}^{\prime} \mathrm{d} \zeta+\int_{0}^{0.5} e^{-\frac{k_{3} z}{\bar{\gamma} \zeta}} \mathcal{A}_{2}^{\prime} \mathrm{d} \zeta\right)
$$

where

$$
\mathcal{A}_{1}^{\prime}=-\frac{[1+(a c-1) \zeta+b c(1-\zeta)]}{(\zeta-1)[(a-b) \zeta+b]^{2}} e^{\frac{a c \zeta}{\zeta-1}}
$$

and

$$
\mathcal{A}_{2}^{\prime}=-\frac{e^{\frac{(a+b) c \zeta}{-1+2 \zeta}}(-1+b c(-1+\zeta)+(2-a c) \zeta)}{(-1+2 \zeta)(b+(a-b) \zeta)^{2}} .
$$

Proof: See Appendix C.

Remark 4: Theorem 3 involves the computation of the integrals with integrands composed of elementary functions. Although such integrals are not in closed-form, they can be easily evaluated numerically by employing standard techniques available in the most common mathematical software packages, such as Matlab, Maple, or Mathematica.

\section{B. Outage Probability}

The OP is defined as the probability that the instantaneous SNR falls below a given threshold $\gamma_{\mathrm{th}}$, i.e.,

$$
P_{\text {out }}\left(\gamma_{\mathrm{th}}\right)=\operatorname{Pr}\left[\gamma<\gamma_{\mathrm{th}}\right]=F_{\gamma}\left(\gamma_{\mathrm{th}}\right) .
$$

We note that the OP at $T_{j}, j=1,2$ is given by

$\operatorname{Pr}\left(\right.$ outage at $\left.T_{j}\right)=\operatorname{Pr}[$ one antenna selected]

$$
\begin{aligned}
\times & \operatorname{Pr}\left[\text { outage at } T_{j} \mid \text { one antenna selected }\right] \\
& +\operatorname{Pr}[\text { two antennas selected }] \\
\times & \operatorname{Pr}\left[\text { outage at } T_{j} \mid \text { two antennas selected }\right] .
\end{aligned}
$$

The OP results are presented in the following corollaries.

Corollary 1: In the joint AS-DE scheme, the tight upper and lower bounds on the OP at $T_{2}$ can be calculated as

$$
P_{\mathrm{out}, \mathrm{ub}}^{\mathrm{ASDE}}\left(\gamma_{\mathrm{th}}\right)=p_{N} F_{\gamma_{1, T_{2}}}\left(\gamma_{\mathrm{th}}\right)+p_{N}^{\prime} \sum_{q=1}^{N-1} F_{\gamma_{T_{2}, \mathrm{bb}}^{\mathrm{ASDE}}}\left(\gamma_{\mathrm{th}}\right),
$$

and

$$
P_{\mathrm{out}, \mathrm{lb}}^{\mathrm{ASE}}\left(\gamma_{\mathrm{th}}\right)=p_{N} F_{\gamma_{1, T_{2}}}\left(\gamma_{\mathrm{th}}\right)+p_{N}^{\prime} \sum_{q=1}^{N-1} F_{\gamma_{T_{2}, \mathrm{ub}}^{\mathrm{ASE}}}\left(\gamma_{\mathrm{th}}\right),
$$

respectively, where $p_{N}=p_{N}^{\prime}=1 / N, F_{\gamma_{1, T_{2}}}\left(\gamma_{\mathrm{th}}\right), F_{\gamma_{T_{2}, \mathrm{ub}}^{\mathrm{ASE}}}\left(\gamma_{\mathrm{th}}\right)$ and $F_{\gamma_{T_{2}, \text { Ib }}^{\mathrm{ASDE}}}\left(\gamma_{\mathrm{th}}\right)$ are presented in Theorem 1 , Theorem 2 and Theorem 3, respectively.

Remark 5: The probabilities $p_{N}$ in (24) and (25) are for the event that one antenna is selected. From (6), it can be seen that among the $N \times N$ pairs $\left(h_{i}, g_{j}\right)(i, j=1,2 \cdots N)$, there are $N$ pairs $\left(h_{l^{*}}, g_{r^{*}}\right)$ with $l^{*}=r^{*}=1, \cdots, N$. We note that each pair is selected with the same probability, since we assume all $h_{i} \mathrm{~s}$ and $g_{j} \mathrm{~s}$ are i.i.d. distributed. Therefore, the probability that one antenna is selected, is $p_{N}=N /(N \times N)=1 / N$. In (24) and (25), $p_{N}^{\prime}$ equals to $(1-1 / N) /(N-1)$, where $1-1 / N$ is the probability that two different antennas have been selected, i.e., $l^{*} \neq r^{*}$, and $1 /(N-1)$ is the probability that $q$ takes a specific value in $\{1, \cdots, N-1\}$.

\section{Diversity Order}

Corollary 2: The proposed joint AS-DE scheme can achieve full diversity, i.e., the diversity order is $N$.

Proof: See Appendix D.

\section{Simulation AND Numerical Results}

In this section, computer simulations are carried out to demonstrate the performance of the joint AS-DE scheme with $\gamma_{\text {th }}=3, P=1$ and $P_{r}=2 P=2$ in all figures, $\Omega=1$ in Figs. 1-3, and $\Omega=1 /(1-d)^{-3}$ in Fig. 4 where $d$ and 3 denote the distance between $T_{1}$ and $R$ and the path-loss exponent, respectively. In addition, numerical results obtained from Corollary 1 are also used to show the accuracy of the developed analytical results. We note that in order to guarantee comparison fairness, the same power consumption used by the joint AS-DE scheme as in the max-min, the max-sum and the max-max schemes is considered. For the max-min and max-sum schemes which select a single antenna at $R$, 


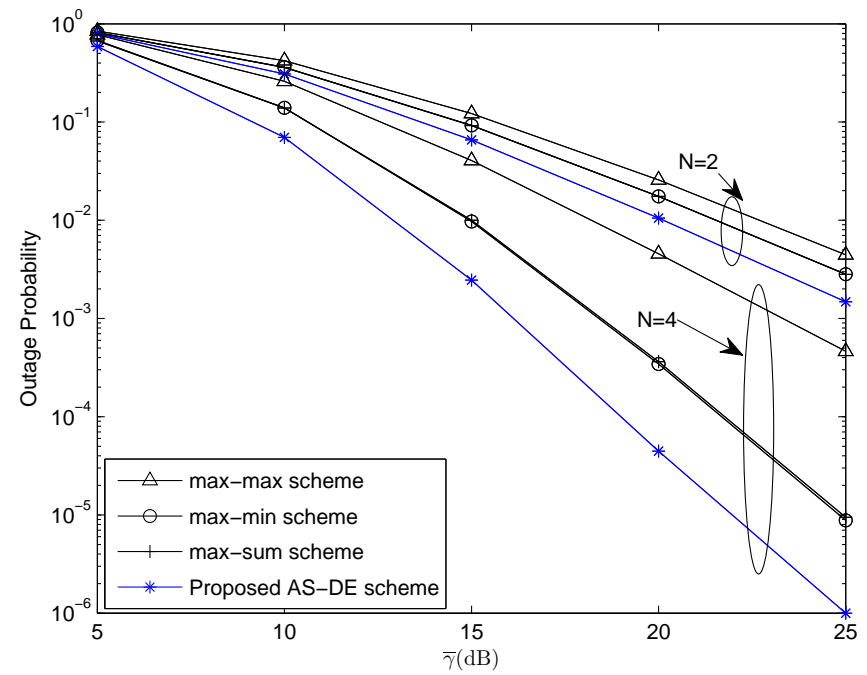

Fig. 1. OP comparisons of different AS schemes under different $\bar{\gamma}$.

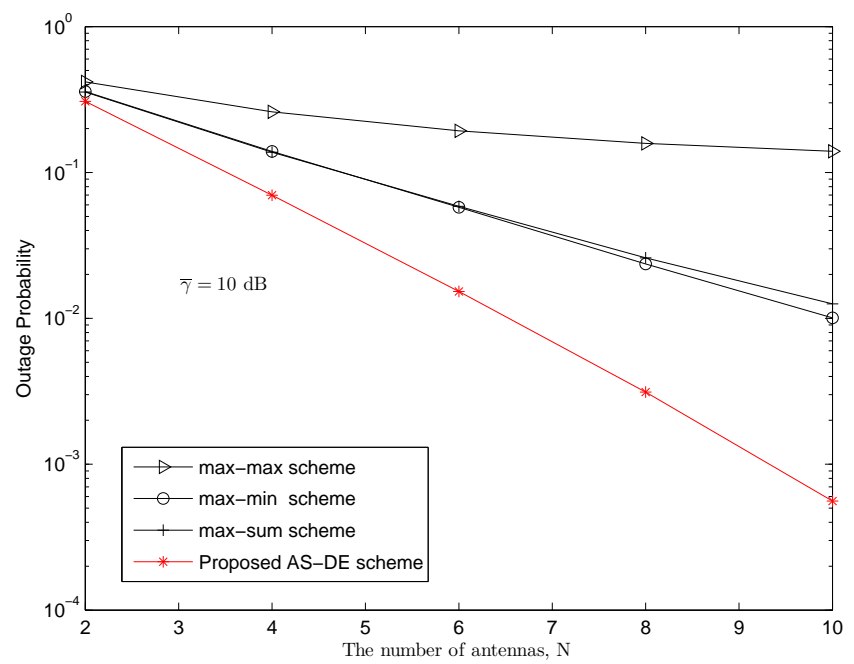

Fig. 2. OP comparisons of different AS schemes under different $N$ and $\bar{\gamma}=10 \mathrm{~dB}$

the whole transmit power at $R$ is $P_{r}=2$. For the proposed AS-DE and max-max schemes, if one antenna is selected, the transmit power of the selected antenna at $R$ is $P_{r}=2$; otherwise, each selected antenna broadcasts the signal with power $P_{r} / 2=1$, indicating that the whole transmit power at $R$ is $P_{r} / 2+P_{r} / 2=2$.

Fig. 1 illustrates the Monte-Carlo simulation results on the OP performance of the joint AS-DE scheme in comparison to the max-min, max-sum, max-max schemes versus $\bar{\gamma}$ under $N=2,4$. It clearly illustrates that under arbitrary $N$ and $\bar{\gamma}$, our proposed joint AS-DE scheme performs much better than the other three AS schemes. For example, when $N=4$ and at $10^{-4} \mathrm{OP}$, the joint AS-DE scheme provides a nearly $3 \mathrm{~dB}$ gain over those of the max-min and max-sum schemes, about a $10 \mathrm{~dB}$ gain over that of the max-max scheme. This result is expected because the joint AS-DE scheme utilizes the

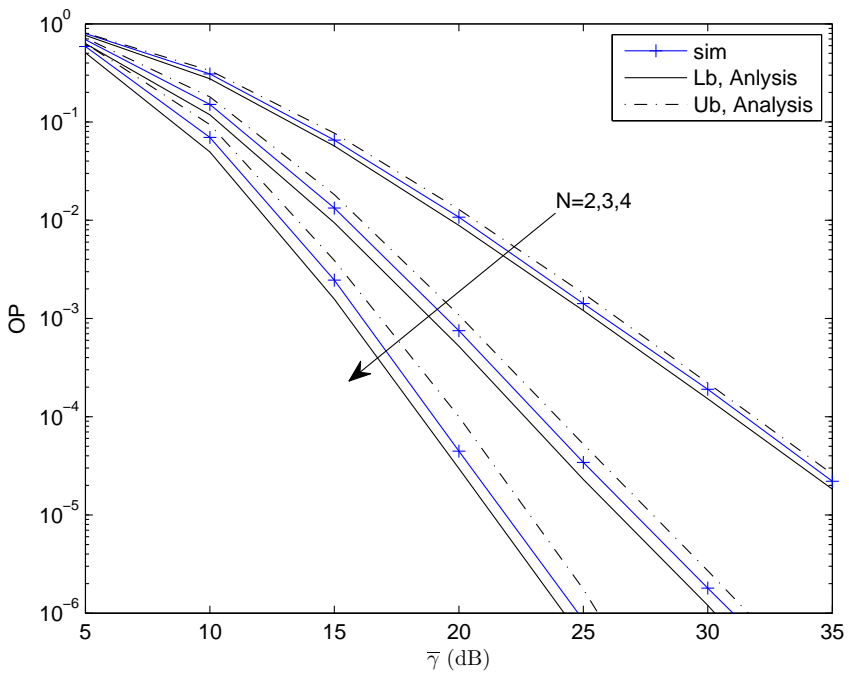

Fig. 3. OP versus $\bar{\gamma}$ under different relay antenna number $N$ in the joint AS-DE scheme.

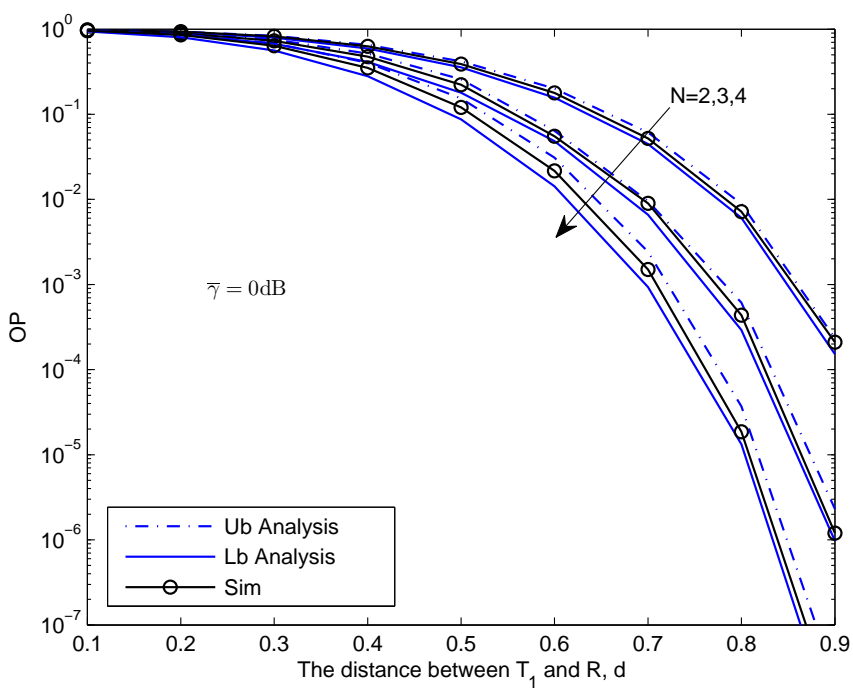

Fig. 4. OP versus the distance between $T_{1} \rightarrow R$ under different relay antenna number $N$ in the joint AS-DE scheme .

"maximum-maximum" and "small-small" channel coefficients combinations, resulting in a larger received SNR. Furthermore, it can also be seen that the joint AS-DE scheme can achieve full diversity as the max-min, max-sum schemes.

Fig. 2 compares the OP performance of the joint AS-DE scheme with the max-min, max-sum and max-max schemes versus the relay antenna number $N$ with $\bar{\gamma}=10 \mathrm{~dB}$. It can be also clearly seen that the joint AS-DE scheme outperforms the other three schemes under an arbitrary $N$. Besides, as the number of relay antennas $N$ increases, the SNR gain that the joint AS-DE scheme achieves over the other three schemes counterpart is further enlarged.

In Fig. 3, the developed analytical results presented in Corollary 1 for the joint AS-DE schemes are compared to the simulation results with $N=2,3$ and 4 . As can be 
observed from the figure, under an arbitrary $N$, the upper and lower bounds on OP are quite close to the simulation counterparts which verifies our analysis. Furthermore, the lower and upper bound outage curves verify our diversity order analysis, indicating that the joint AS-DE scheme can achieve full diversity.

In Fig. 4, the impact of the relay location on OP for $T_{2}$ is studied in the joint AS-DE scheme. Specifically, the OP is plotted against the distance $d$ between $T_{1}$ and $R$ by modeling the path-loss dependent parameters $\Omega=1 /(1-d)^{-3}$. As can be observed, the OP performance at $T_{2}$ improves as the relay station gets close to $T_{2}$. Besides, the lower and upper bounds we derived are quite tight under an arbitrary $d$ indicating the accuracy of our analysis.

\section{CONCLUSION}

In this paper, we proposed an efficient transmission scheme for multi-antenna AF TWRNs, termed as the joint AS-DE scheme. Particularly, the joint AS-DE scheme utilized the antenna selection criterion in the max-max scheme along with data exchange to transmit data through the links characterized by "maximum-maximum" and "small-small" channel coefficient combinations. We presented the tight lower and upper bounds on the OP for the proposed scheme. Furthermore, our analysis revealed that the joint AS-DE scheme can achieve full diversity. Finally, analysis and simulation results showed that under the same time slots and power consumption, the joint AS-DE scheme outperforms the existing schemes, i.e., the max-min, max-sum and max-max ones. For example, when $N=4$ and at $10^{-4} \mathrm{OP}$, the joint AS-DE scheme provides a nearly $3 \mathrm{~dB}$ gain when compared with the max-min and maxsum schemes, and about a $10 \mathrm{~dB}$ gain when compared with the max-max scheme.

\section{APPENDIX A \\ PROOF OF THEOREM 1}

Since all links experience i.i.d. Rayleigh fading, the PDF and CDF of the instantaneous SNR of any links, $\gamma_{i}^{l}$ or $\gamma_{i}^{r}$ follow that

$$
f_{\gamma_{i}^{l}}(x)=\frac{1}{\bar{\gamma}} e^{-\frac{x}{\bar{\gamma}}}, \quad F_{\gamma_{i}^{l}}(x)=1-e^{-\frac{x}{\bar{\gamma}}} .
$$

Based on the order statistics in [20], we have

$$
F_{\gamma_{(N)}^{l}}(x)=\left(F_{\gamma_{i}^{l}}(x)\right)^{N}=\sum_{1, k_{1}} e^{-\frac{k_{1} x}{\gamma}} .
$$

The PDF of $\gamma_{(N)}^{l}$ can be obtained as,

$$
f_{\gamma_{(N)}^{l}}(x)=\sum_{2, k_{2}} e^{-\frac{k_{2} x}{\gamma}} .
$$

Similarly, we have

$$
f_{\gamma_{(N)}^{r}}(y)=\sum_{2, k_{2}} e^{-\frac{k_{2} y}{\gamma}} .
$$

From (7), $F_{\gamma_{1, T_{2}}}(z)$ can be expressed as

$$
F_{\gamma_{1, T_{2}}}(z)=\int_{0}^{\infty} \operatorname{Pr}\left(\gamma_{(N)}^{l} \leq z+\frac{2 c z}{2 y}\right) f_{\gamma_{(N)}^{r}}(y) \mathrm{d} y .
$$

Utilizing [12, Eq.(3.471.9)], Theorem 1 can be achieved.

\section{APPENDIX B}

PROOF OF THEOREM 2

We will firstly study the distribution of $\theta=\gamma_{(q)}^{r}+\gamma_{(N)}^{r}$, and then the distribution of $u=\theta /(\theta+c)$. Finally, the distribution of $\gamma_{T_{2}, \mathrm{ub}}^{\mathrm{ASE}}=\gamma_{(N)}^{l} u$ will be obtained.

Now, let's study the CDF of $\theta=\gamma_{(q)}^{r}+\gamma_{(N)}^{r}$. Based on the order statistics in [20], the joint PDF of $\gamma_{(q)}^{r}$ and $\gamma_{(N)}^{r}$, $1 \leq q<N$, is

$$
f_{\gamma_{(q)}^{r}, \gamma_{(N)}^{r}}(s, v)=\widehat{\sum_{3, k_{1}, k_{2}}} e^{-b s} e^{-a v},
$$

for $0<s<v<\infty$

Therefore, the $\mathrm{CDF}$ of $\theta$, i.e., $F_{\theta}(\vartheta)$, follows that

$$
\begin{aligned}
F_{\theta}(\vartheta) & =\int_{0}^{\frac{\vartheta}{2}} \int_{s}^{\vartheta-s} f_{\gamma_{(q)}^{r}, \gamma_{(N)}^{r}}(s, v) \mathrm{d} v \mathrm{~d} s \\
& =\sum_{3, k_{1}, k_{2}} \frac{1}{a} \int_{0}^{\vartheta / 2}\left[e^{-(a+b) s}-e^{(a-b) s-a \vartheta}\right] \mathrm{d} s .
\end{aligned}
$$

Utilizing [12, Eq. (2.311)], we have

$F_{\theta}(\vartheta)=\left\{\begin{array}{l}-\widehat{\sum_{3, k_{1}, k_{2}}} \frac{1}{a}\left[\frac{\vartheta}{2} e^{-a \vartheta}+\frac{e^{-\frac{(a+b) \vartheta}{2}}}{a+b}\right], a-b=0, \\ \widehat{\sum_{3, k_{1}, k_{2}} \frac{1}{a}}\left[\frac{e^{-a \vartheta}-e^{-\frac{(a+b) \vartheta}{2}}}{a-b}+\frac{1-e^{-\frac{(a+b) \vartheta}{2}}}{a+b}\right], a-b \neq 0 .\end{array}\right.$

From $u=\theta /(\theta+c)$, we have $\theta=g(u)$, where $g(u)=$ $\frac{c u}{1-u}$. Therefore, $f_{u}(u)=f_{\theta}(g(u))\left|g^{\prime}(u)\right|$, where $g^{\prime}(u)$ denotes the derivative of $g(u)$.

Taking the derivative of (B-3), we can obtain the PDF of $\theta$, i.e. $f_{\theta}(\vartheta)$. And then, $f_{u}(u)$ can be obtained as follows,

$f_{u}(u)= \begin{cases}\widehat{\sum_{3, k_{1}, k_{2}}} \frac{c^{\prime 2} u}{2(1-u)^{3}} e^{-\frac{a c u}{1-u}}, & a=b \\ \sum_{3, k_{1}, k_{2}} \frac{c^{\prime}}{(b-a)(1-u)^{2}}\left(e^{-\frac{a c u}{1-u}}-e^{-\frac{(a+b) c u}{2(1-u)}}\right), & a \neq b \\ 0, & u>1 .\end{cases}$

The CDF of $\gamma_{T_{2} \text {,ub }}^{\mathrm{ASDE}}$ is given as follows

$$
F_{\gamma_{T_{2}, \text { ub }}^{\text {ASDE }}}(z)=\int_{-\infty}^{\infty} F_{\gamma_{(N)}^{l}}\left(\frac{z}{u}\right) f_{u}(u) \mathrm{d} u .
$$

Substituting (A-1) and (B-4) into (B-5), with the aid of [12, Eq. (3.351.2)] and [12, Eq. (3.471.9)], Theorem 2 is deduced.

\section{APPENDIX C \\ PROOF OF THEOREM 3}

We firstly study the distribution of $\varepsilon=$ $\gamma_{(N)}^{r} /\left(\gamma_{(q)}^{r}+\gamma_{(N)}^{r}+c\right)$, and then the distribution of $\gamma_{T_{2}, \mathrm{lb}}^{\mathrm{ASDE}}=\varepsilon \gamma_{(N)}^{l}$.

The $\mathrm{CDF}$ of $\varepsilon$ is given as

$$
F_{\varepsilon}(\zeta)=\operatorname{Pr}\left(\frac{\gamma_{(N)}^{r}}{\gamma_{(q)}^{r}+\gamma_{(N)}^{r}+c} \leq \zeta\right) .
$$


When $1 / 2 \leq \zeta \leq 1$, substituting (B-1) into (C-1), we have

$$
\begin{aligned}
F_{\varepsilon}(\zeta) & =\widehat{\sum_{3, k_{1}, k_{2}}} \int_{0}^{\infty} \int_{s}^{\frac{(s+c) \zeta}{1-\zeta}} e^{-a v} e^{-b s} \mathrm{~d} v \mathrm{~d} s \\
& =\widehat{\sum_{3, k_{1}, k_{2}}} \underbrace{\frac{1}{a(a+b)}+\frac{(-1+\zeta) e^{\frac{a c \zeta}{-1+\zeta}}}{a(b+(a-b) \zeta)}}_{\mathcal{A}_{1}} .
\end{aligned}
$$

When $0 \leq \zeta \leq 1 / 2$, (C-1) becomes

$$
\begin{aligned}
F_{\varepsilon}(\zeta) & =\widehat{\sum_{3, k_{1}, k_{2}}} \int_{0}^{\frac{c \zeta}{1-2 \zeta}} \int_{s}^{\frac{(s+c) \zeta}{1-\zeta}} e^{-a v} e^{-b s} \mathrm{~d} v \mathrm{~d} s \\
& =\widehat{\sum_{3, k_{1}, k_{2}}}(\mathcal{A}_{1}+\underbrace{\frac{(-1+\zeta) e^{\frac{(a+b) c \zeta}{-1+2 \zeta}}}{a(-b+(b-a) \zeta)}-\frac{e^{\frac{(a+b) c \zeta}{-1+2 \zeta}}}{a(a+b)}}_{\mathcal{A}_{2}}) .
\end{aligned}
$$

Therefore, the PDF of $\varepsilon$ can be obtained as,

$$
f_{\varepsilon}(\zeta)= \begin{cases}\widehat{\sum_{3, k_{1}, k_{2}}} \mathcal{A}_{1}^{\prime}, & 1 / 2<\zeta<1, \\ \sum_{3, k_{1}, k_{2}}\left(\mathcal{A}_{1}^{\prime}+\mathcal{A}_{2}^{\prime}\right), & 0<\zeta \leq 1 / 2, \\ 0, & \text { others. }\end{cases}
$$

where $\mathcal{A}_{1}^{\prime}$ and $\mathcal{A}_{2}^{\prime}$ are given in (21) and (22), respectively. Therefore, the CDF of $\gamma_{T_{2}, \mathrm{lb}}^{\mathrm{ASDE}}$, i.e., $F_{\gamma_{T_{2}, \mathrm{lb}}^{\mathrm{ASDE}}}\left(\gamma_{t h}\right)$, is

$$
F_{\gamma_{T_{2}, \mathrm{bl}}^{\mathrm{ASDE}}}\left(\gamma_{t h}\right)=\int_{0}^{\infty} F_{\gamma_{(N)}^{l}}\left(\frac{\gamma_{t h}}{\zeta}\right) f_{\varepsilon}(\zeta) \mathrm{d} \zeta
$$

Substituting (A-1) and (C-2) into (C-3), Theorem 3 can be reached.

\section{APPENDIX D}

\section{PROOF OF COROLLARY 2}

The following facts are utilized, i.e., $\lim _{x \rightarrow 0} e^{-x}=1-x$, $\lim _{x \rightarrow 0} K_{1}(x)=1 / x$ and $\lim _{x \rightarrow 0} K_{2}(x)=2 / x^{2}$.

Recalling Theorem 1, (18) can be re-expressed as,

$$
\begin{aligned}
F_{\gamma_{1, T_{2}}}(z) & =1+\sum_{k_{2}=1}^{N}\left(\begin{array}{l}
N \\
k_{2}
\end{array}\right)(-1)^{k_{2}+1} \sum_{k_{1}=1}^{N}\left(\begin{array}{l}
N \\
k_{1}
\end{array}\right)(-1)^{k_{1}} e^{-\frac{k_{1} z}{\bar{\gamma}}} \\
& =1+\left[\left(1-e^{-\frac{z}{\bar{\gamma}}}\right)^{N}-1\right] .
\end{aligned}
$$

When $\bar{\gamma} \rightarrow \infty$, we have

$$
F_{\gamma_{1, T_{2}}}(z) \stackrel{\bar{\gamma} \rightarrow \infty}{=}\left(\frac{z}{\bar{\gamma}}\right)^{N}
$$

Utilizing (A-1), (C-3) can be rewritten as

$$
\begin{aligned}
F_{\gamma_{T_{2}, \mathrm{lb}}^{\mathrm{ASDE}}}(z) & =\int_{0}^{\infty}\left(1-e^{-\frac{z}{\bar{\gamma} \zeta}}\right)^{N} f_{\varepsilon}(\zeta) \mathrm{d} \zeta \\
& \stackrel{\bar{\gamma} \rightarrow \infty}{=}\left(\frac{z}{\bar{\gamma}}\right)^{N} \int_{0}^{\infty} \frac{1}{\zeta^{N}} f_{\varepsilon}(\zeta) \mathrm{d} \zeta .
\end{aligned}
$$

We note that $\int_{0}^{\infty} \frac{1}{\zeta^{N}} f_{\varepsilon}(\zeta) \mathrm{d} \zeta$ is a constant, which is independent of $\bar{\gamma}$.

Based on (D-1) and (D-2), at the high SNR regions, (24) can be asymptotically approximated by

$$
P_{\text {out,ub }}^{\mathrm{ASDE}}\left(\gamma_{\mathrm{th}}\right) \stackrel{\bar{\gamma} \rightarrow \infty}{=}\left[\frac{1}{N}+\frac{1}{N} \sum_{q=1}^{N-1} \int_{0}^{\infty} \frac{1}{\zeta^{N}} f_{\varepsilon}(\zeta) \mathrm{d} \zeta\right]\left(\frac{\gamma_{\mathrm{th}}}{\bar{\gamma}}\right)^{N} .
$$

Recalling Theorem $2, \mathcal{L}_{1}$ in (19) can be deduced as, when $\bar{\gamma} \rightarrow \infty$

$$
\begin{aligned}
\mathcal{L}_{1} & \stackrel{\bar{\gamma} \rightarrow \infty}{=} \sum_{3, k_{1}, k_{2}}\left[\frac{1}{a(a+b)}+\frac{1}{2 a^{2}} \sum_{k_{3}=1}^{N}\left(\begin{array}{l}
N \\
k_{3}
\end{array}\right)(-1)^{k_{3}} e^{-\frac{k_{3} z}{\bar{\gamma}}}\right] \\
& =\widehat{\sum_{3, k_{1}, k_{2}}}\left\{\frac{1}{a(a+b)}+\frac{1}{2 a^{2}}\left[\left(1-e^{-\frac{z}{\bar{\gamma}}}\right)^{N}-1\right]\right\} \\
& =\sum_{3, k_{1}, k_{2}} \frac{1}{a(a+b)}\left(\frac{z}{\bar{\gamma}}\right)^{N} .
\end{aligned}
$$

Similarly, $\mathcal{L}_{2}$ in (19) can be obtained as follows, when $\bar{\gamma} \rightarrow \infty$,

$$
\mathcal{L}_{2} \stackrel{\bar{\gamma} \rightarrow \infty}{=} \sum_{3, k_{1}, k_{2}} \frac{1}{a(a+b)}\left(\frac{z}{\bar{\gamma}}\right)^{N}
$$

Utilizing (D-4) and (D-5), when $\bar{\gamma} \rightarrow \infty$, (19) is deduced as,

$$
F_{\gamma_{T_{2}, \mathrm{ub}}^{\mathrm{ASDE}}}(z) \stackrel{\bar{\gamma} \rightarrow \infty}{=} \widehat{\sum}_{3, k_{1}, k_{2}} \frac{1}{a(a+b)}\left(\frac{z}{\bar{\gamma}}\right)^{N}
$$

From (D-1) and (D-6), at the high SNR regions, (25) can be asymptotically approximated by

$$
P_{\mathrm{out}, \mathrm{lb}}^{\mathrm{ASDE}}\left(\gamma_{\mathrm{th}}\right) \stackrel{\bar{\gamma} \rightarrow \infty}{=}\left[\frac{1}{N}+\frac{1}{N} \sum_{q=1}^{N-1} \widehat{\sum_{3, k_{1}, k_{2}}} \frac{1}{a(a+b)}\right]\left(\frac{\gamma_{\mathrm{th}}}{\bar{\gamma}}\right)^{N} .
$$

Finally, Corollary 2 is proved from (D-7) and (D-3).

\section{REFERENCES}

[1] B. Rankov and A. Wittneben, "Spectral efficient protocols for halfduplex fading relay channels," IEEE J. Sel. Areas Commun., vol. 25, no. 2, pp. 379-389, Feb. 2007.

[2] J. Yang, P. Fan, T. Duong, and X. Lei, "Exact performance of two-way AF relaying in Nakagami- $m$ fading environment," IEEE Trans. Wireless Commun., vol. 10, no. 3, pp. 980-987, Mar. 2011.

[3] H. Gao, T. Lv, S. Zhang, C. Yuen, and S. Yang, "Zero-forcing based MIMO two-way relay with relay antenna selection: Transmission scheme and diversity analysis," IEEE Trans. Wireless Commun., vol. 11, no. 12, pp. 4426-4437, Dec. 2012.

[4] I. Krikidis, S. Sasaki, S. Timotheou, and Z. Ding, "A low complexity antenna switching for joint wireless information and energy transfer in MIMO relay channels," IEEE Trans. on Commun., vol. 62, no. 5, pp. 1577-1587, May 2014.

[5] P. Zhang, S. Chen, and L. Hanzo, "Two-tier channel estimation aided near-capacity MIMO transceivers relying on norm-based joint transmit and receive antenna selection," IEEE Trans. Wireless Commun., vol. 14, no. 1, pp. 122-137, Jan. 2015.

[6] C. Jiang and L. Cimini, "Antenna selection for energy-efficient MIMO transmission," IEEE Wireless Commun. Lett., vol. 1, no. 6, pp. 577-580, Dec. 2012. 
[7] G. Amarasuriya, C. Tellambura, and M. Ardakani, "Two-way amplifyand-forward MIMO relay networks with antenna selection," in Proc. IEEE Globecom, Houston, USA, Dec. 2011, pp. 1-5.

[8] K. Yang, N. Yang, C. Xing, and J. Wu, "Relay antenna selection in MIMO two-way relay networks over Nakagami- $m$ fading channels," IEEE Trans. Veh. Technol., vol. 63, no. 5, pp. 2349-2362, Jun. 2014.

[9] G. Amarasuriya, C. Tellambura, and M. Ardakani, "Two-way amplifyand-forward multiple-input multiple-output relay networks with antenna selection," IEEE J. Sel. Areas Commun., vol. 30, no. 8, pp. 1513-1529, Sep. 2012

[10] M. Eslamifar, W. H. Chin, C. Yuen, and Y. L. Guan, "Performance analysis of two-way multiple-antenna relaying with network coding," in Proc. IEEE VTC-Fall, Anchorage, Alaska, Sep. 2009, pp. 1-5.

[11] Y. Li, R. H. Y. Louie, and B. Vucetic, "Relay selection with network coding in two-way relay channels," IEEE Trans. Wireless Commun., vol. 59, no. 9, pp. 4489-4499, Nov. 2010.

[12] I. S. Gradshteyn and I. M. Ryzhik, Table of Integrals, Series, and Products, 6th ed. San Diego, CA: Academic Press, 2000.

[13] T. Cui, F. Gao, T. Ho, and A. Nallanathan, "Distributed space-time coding for two-way wireless relay networks," IEEE Trans. Signal Process., vol. 57, no. 2, pp. 658-671, Feb. 2009.

[14] W. Wang, S. Jin, X. Gao, K.-K. Wong, and M. McKay, "Power allocation strategies for distributed space-time codes in two-way relay networks," IEEE Trans. Signal Process., vol. 58, no. 10, pp. 5331-5339, 2010.

[15] B. Maham, A. Hjorungnes, and G. Abreu, "Distributed gabba space time codes in amplify-and-forward relay networks," IEEE Trans. Wireless Commun., vol. 8, no. 4, pp. 2036-2045, Apr. 2009.

[16] Y. Jing and H. Jafarkhani, "Using orthogonal and quasi-orthogonal designs in wireless relay networks," IEEE Trans. Inf. Theory, vol. 53, no. 11, pp. 4106-4118, Nov. 2007.

[17] M. D. Renzo, H. Haas, and P. M. Grant, "Spatial modulation for multiple-antenna wireless systems: a survey," IEEE Commun. Mag., vol. 49, no. 12, pp. 182-191, Dec. 2011.

[18] V. Barousis, A. Kanatas, A. Kalis, and C. Papadias, "A stochastic beamforming algorithm for ESPAR antennas," IEEE Antennas Wireless Propag. Lett., vol. 7, no. 1, pp. 745-748, Aug. 2008.

[19] R. Qian, M. Sellathurai, and D. Wilcox, "A study on MVDR beamforming applied to an ESPAR antenna," IEEE Signal Process. Lett., vol. 22, no. 1, pp. 67-70, Jan. 2015.

[20] H. David, Order statistics. New York: Wiley, 1980.

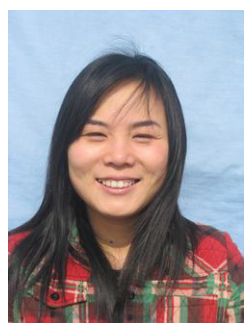

Jing Yang was born in Shandong, China, in 1982 She received her $\mathrm{Ph}$. D. degree in communications and information systems from Southwest Jiaotong University, Chengdu, in 2013. Since Mar. 2013, she has been with the School of Information Engineering, Yangzhou University, as a Associate Professor. From Sep. 2015 to Feb. 2016, she was a Visiting Scholar in University of Victoria, Canada. Her research interests include $5 \mathrm{G}$ networks, cooperative and energy harvesting networks, physical layer security and massive MIMO.

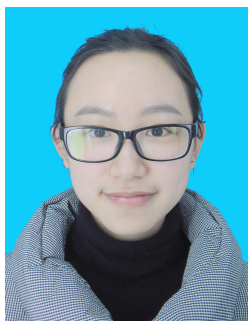

Lei Chen was born in Jiangsu, China, in 1991. She received the B.S. degree in communication engineering from Yangzhou University, Jiangsu, in 2009. She is currently working towards her M.S. degree in the School of Information Engineering, Yangzhou University. Her research interests are in cooperative relaying communications, cognitive radio networks, physical layer security.
Xianfu Lei was born in 1981. He received the Ph.D. degree in communication and information systems from Southwest Jiaotong University, China, in 2012. From 2012 to 2014, he was a Research Fellow with the Department of Electrical \& Computer Engineering, Utah State University, USA. Since 2015, he has been an Associate Professor with Southwest Jiaotong University. His research interests include 5G communications, cooperative communications, and energy harvesting. He has authored over 70 research papers on these topics. He received the Exemplary Reviewer Certificate of the IEEE Communications Letters and an Exemplary Reviewer Certificate of the IEEE Wireless Communications Letters in 2013. He has been a TPC Chair of several international conferences and workshops, including the most recently the IEEE ICC18 Symposium on Ad-Hoc and Sensor Networking. He currently serves as an Editor of the IEEE COMMUNICATIONS LETTERS and the IEEE ACCESS. He has served as a Guest Editor of the IEEE JOURNAL ON SELECTED AREAS IN COMMUNICATIONS.

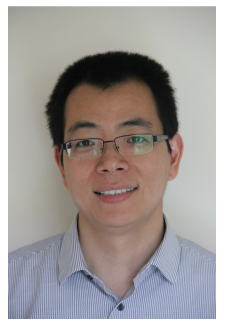

Zhiguo Ding (S'03-M'05) received his B.Eng in Electrical Engineering from the Beijing University of Posts and Telecommunications in 2000, and the $\mathrm{Ph} . \mathrm{D}$ degree in Electrical Engineering from Imperial College London in 2005. From Jul. 2005 to Aug. 2014, he was working in Queen's University Belfast, Imperial College and Newcastle University. Since Sept. 2014, he has been with Lancaster University as a Chair Professor. From Oct. 2012 to Sept. 2019, he has also been an academic visitor in Princeton University. Dr Ding' research interests are 5G networks, game theory, cooperative and energy harvesting networks and statistical signal processing. He is serving as an Editor for IEEE Transactions on Communications, IEEE Transactions on Vehicular Technology, and Journal of Wireless Communications and Mobile Computing, and was an Editor for IEEE Wireless Communication Letters, IEEE Communication Letters from 2013 to 2016. He received the best paper award in IET Comm. Conf. on Wireless, Mobile and Computing, 2009, IEEE Communication Letter Exemplary Reviewer 2012, and the EU Marie Curie Fellowship 2012-2014.

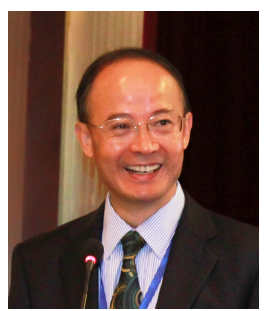

PingzhiFan (M'93-SM'99-F'15) received his $\mathrm{PhD}$ degree in Electronic Engineering from the Hull University, UK. He is currently a professor and director of the institute of mobile communications, Southwest Jiaotong University, China. He is a recipient of the UK ORS Award, the Outstanding Young Scientist Award by NSFC, and the chief scientist of a national 973 research project. He served as general chair or TPC chair of a number of international conferences, and is the guest editor-in-chief, guest editor or editorial member of several international journals. He is the founding chair of IEEE VTS BJ Chapter and IEEE ComSoc CD Chapter, the founding chair of IEEE Chengdu Section. He also served as a board member of IEEE Region 10, IET(IEE) Council and IET AsiaPacific Region. He has over 200 research papers published in various academic English journals (IEEE/IEE/IEICE, etc), and 8 books (incl. edited), and is the inventor of 22 granted patents. His research interests include high mobility wireless communications, $5 \mathrm{G}$ technologies, wireless networks for big data, signal design \& coding, etc. He is an IEEE VTS Distinguished Lecturer (2015-2019), and a fellow of IEEE, IET, CIE and CIC. 


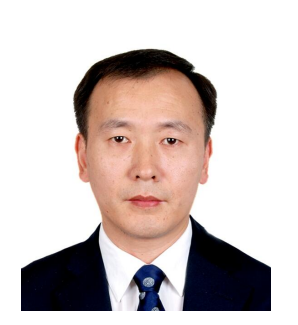

XiqiGao (S'92-AM'96-M'02-SM'07-F'15) received the Ph.D. degree in electrical engineering from Southeast University, Nanjing, China, in 1997. He joined the Department of Radio Engineering, Southeast University, in April 1992. Since May 2001, he has been a professor of information systems and communications. From September 1999 to August 2000, he was a visiting scholar at Massachusetts Institute of Technology, Cambridge, and Boston University, Boston, MA. From August 2007 to July 2008, he visited the Darmstadt University of Technology, Darmstadt, Germany, as a Humboldt scholar. His current research interests include broadband multicarrier communications, MIMO wireless communications, channel estimation and turbo equalization, and multirate signal processing for wireless communications. From 2007 to 2012, he served as an Editor for the IEEE Transactions on Wireless Communications. From 2009 to 2013, he served as an Associate Editor for the IEEE Transactions on Signal Processing. From 2015 to 2017, he served as an Editor for the IEEE Transactions on Communications. Dr. Gao received the Science and Technology Awards of the State Education Ministry of China in 1998, 2006 and 2009, the National Technological Invention Award of China in 2011, and the 2011 IEEE Communications Society Stephen O. Rice Prize Paper Award in the field of communications theory. 